\title{
Serum soluble cluster of differentiation 163 (sCD163), matrix metalloproteinase 9 (MMP9) and cytokeratin 18 (CK18) levels as biomarkers for liver fibrosis in Egyptian chronic hepatitis $\mathrm{C}$ patients
}

\author{
S. M. Mostafa ${ }^{1}$, R. H. Mohamed ${ }^{2}$, A. M. Abdel Baki ${ }^{1}$, E. M. Abd El Azeem², E. M. Saleh ${ }^{2}$ \\ ${ }^{1}$ Biochemistry Department, National Hepatology and Tropical Medicine Research Institute, Egypt. \\ ${ }^{2}$ Biochemistry Department, Faculty of Science, Ain Shams University, Cairo, Egypt.
}

\section{ARTICLE INFO}

Received 21 June 2021

Accepted 11 October 2021

Keywords

HCV,

Fibrosis,

sCD163,

MMP9,

CK18.

Correspondence

S. M. Mostafa

E-mail

shaimaaa.othman83@gmail.com

\section{ABSTRACT}

Liver fibrosis is a pathological disease in which extracellular matrix proteins, such as collagen, build up in the liver, causing tissue remodeling and the creation of persistent scar tissue. It has been associated to HCV infection, which is a leading cause of liver cirrhosis and hepatocellular carcinoma. These complications are always identified in the late stages and almost through liver biopsy, making the chances for diagnosis and treatment challenging. As a result, the objective of this study is to evaluate the levels of SCD163, MMP9, and CK18 as noninvasive biomarkers for different grades of liver fibrosis in Egyptian chronic hepatitis C patients. A total of 100 subjects were divided into four groups for this investigation. Group 1 consisted of healthy control subjects (Control group). Patients with HCV infection were divided into three groups based on their fibrosis grade: Group 2 (no liver fibrosis, F0), Group 3 (liver fibrosis, F1-F2), and Group 4 (liver fibrosis, F3-F4). The ELISA technique was used to assess the levels of direct serum indicators SCD163, MMP9, CK18, and AFP. A complete blood count, serum ALT, AST, albumin, total bilirubin, and INR were measured. In addition, as indirect biomarkers, the FIB-4 score and the AST/ALT Ratio (AAR) were reported. Results indicated that the levels of sCD163, CK18, and AFP were increased significantly in all HCV infected groups, compared to controls, with the highest levels in the advanced groups. MMP9 was decreased in low grade fibrotic HCV groups, but significantly increased in advanced liver fibrosis group. FIB-4 and AAR showed a significant increase in all HCV patients, compared to control group. From the obtained data, the performance of MMP9 was the most accurate direct blood biomarker for evaluating the advanced fibrosis (Group 4). In addition, CK18 and SCD136 showed promising results. Finally, the triple biomarker panel could be considered as a useful disease progression biomarker.

\section{Introduction}

Hepatitis C virus (HCV) infects more than 170 million people globally, with $70 \%$ of those infected become long-term carriers. Only a minority of infected people eliminate the virus spontaneously, while $30 \%$ to $60 \%$ develop chronic liver illness, with a significant proportion developing chronic liver disease and a substantial percentage develops cirrhosis, or even hepatocellular carcinoma (HCC) ${ }^{[1]}$. Egypt has a high prevalence of HCV infection, with an estimated 8-10 with $\mathrm{HCV}(14.7 \%)^{[2]}$. 
Chronic HCV infection is the leading cause to liver inflammation. If untreated, fibrosis, cirrhosis, and eventually hepatocellular carcinoma may develop [3], which is associated with persistent uncontrolled inflammation. In turn, this activates numerous immune cells such as infiltrating macrophages, T-lymphocytes, neutrophils, and dendritic cells ${ }^{[4]}$.

Hepatic stellate cells, which are the primary source of myofibroblasts in the liver, are activated by inflammatory cells, resulting in the formation of nodules of regenerating hepatocytes. Chronic HCV infection is typically characterized by slowly progressive hepatic fibrosis from stage 0 (no fibrosis) to stage 4 (cirrhosis). Liver fibrosis develops when scar tissue builds up in the organ as a result of repeated or longterm damage or inflammation ${ }^{[5]}$.

Fibrosis stage is assessed after taking a liver biopsy by using different scoring systems such as METAVIR (stages I-IV), which assigns a score for activity or predicts of how fibrosis will progress. Furthermore, Ishak score (stages I-V) is used for fibrosis grading, which assigns numbers to the severity of the necroinflammatory characteristics (interface hepatitis, confluent necrosis, parenchymal injury, and portal inflammation) ${ }^{[6]}$. Higher stages of fibrosis have been related to the progression to decompensated cirrhosis. The need for liver transplantation and liver-related mortality are both evident in HCV infection [7]. Assessment of hepatic fibrosis in patients with persistent $\mathrm{HCV}$ infection is considered a relevant part of patient care and decision-making ${ }^{[3]}$.

For the assessment of liver fibrosis, liver biopsy has traditionally been regarded as the gold standard method. It is an invasive procedure that causes pain, bleeding, leakage of bile from the liver or gallbladder, and other serious complications. Besides, sampling error is possible, particularly when tiny biopsies are evaluated. In addition, variation in biopsies interpretation may also result in incorrect staging ${ }^{[8]}$. On the other hand, transient elastography (FibroScan) is a non-invasive for detecting liver stiffness and assessing hepatic fibrosis and cirrhosis in patients with chronic liver disease, especially, for those who are chronically infected with HCV. However, the method requires a trained staff, and valid measurements from obese patients and patients with ascites may be difficult to obtain ${ }^{[9]}$.
The detection of blood biomarkers has been used as one of several diagnostic methods for evaluating liver fibrosis ${ }^{[10]}$. Surprisingly, some novel experimental markers may be promising tools for improving the noninvasive diagnostic ability of liver fibrosis and cirrhosis. Blood markers are less expensive non-invasive tests that can be used more widely ${ }^{[11]}$. Many of these serum markers are either enzymes, such as alanine aminotransferase (ALT), aspartate aminotransferase (AST), or secreted molecules, such as bilirubin, alphafetoprotein (AFP), alpha-2-macroglobulin, haptoglobin, and apolipoprotein A1. Biomarkers panels have been established in recent years for clinical use. While some of these markers, or combinations of them, are now routinely used in clinical practice, their predictive relevance remains unclear ${ }^{[12]}$.

One of the most intensively studied biomarkers is cluster of differentiation 163 (CD163). It is a hemoglobin-haptoglobin scavenger receptor that exerts its main biological function through the elimination of hemoglobin-haptoglobin complexes during hemolysis [13]. The scavenger receptor CD163 is expressed by macrophages and monocytes and has great interest since its activation by various stimuli induces its shedding into the circulation to give the soluble form (sCD163) which is present in the plasma. Thus, quantifying concentrations of this soluble biomarker may be of promising clinical relevance in estimating the severity of inflammation and fibrosis ${ }^{[14]}$. Jessica et al. ${ }^{[15]}$ reported that SCD163 would associate strongly with hepatic inflammation and fibrosis as a result of its relative specificity for the activation of Kupffer cells, which have a key role in liver injury and inflammation also by producing a large number of matrix metalloproteinases (MMPs).

MMPs are zinc-dependent peptidases that are considered as the principal effectors of extracellular matrix (ECM) protein degradation, maintaining the homeostasis between fibrogenesis and fibrolytic processes in the liver ${ }^{[16]}$. They are not only responsible for ECM degradation, but also shed cell membrane proteins, and cleave diverse bioactive mediators such as cytokines and chemokines, modulating their activity either by direct cleavage, or releasing them from ECM bound stocks ${ }^{[17]}$. Several studies have shown that MMPs play an important role in the pathogenesis of liver fibrosis, and their presence in the advanced stages of fibrosis highlights their role in diagnostic area ${ }^{[18]}$. 
Cytokeratins are proteins of keratin-containing intermediate filaments found in the cytoskeleton of epithelial tissue. They are expressed primarily in the epithelia of the liver, intestine, and exocrine pancreas ${ }^{[19]}$. The level of caspase cleaved fragmented CK18 in serum reflects the degree of apoptosis and has been shown to be an independent predictor of liver damage progression. Many studies have evaluated CK18 as a marker for fibrosis, where it is expressed in hepatocytes as a result of apoptosis and necrosis, which cause inflammation and liver fibrosis ${ }^{[20]}$.

In light of this previous data, the current study was conducted to evaluate the potential of non-invasive biomarkers, such as sCD163, MMP9, and CK18, as alternative markers for the diagnosis of HCV Egyptian patients with different grades of liver fibrosis. Moreover, using these markers to discriminate between different stages of fibrosis in patients chronically infected with HCV.

\section{Material and methods}

\subsection{Patients}

This prospective study included 100 subjects divided into 4 groups who recruited from National Hepatology and Tropical Medicine Research Institute. Group 1 consisted of 25 healthy control subjects (Control group). In addition, $75 \mathrm{HCV}$ infected patients divided into three groups based on their fibrosis grade: Group 2 (no liver fibrosis F0), Group 3 (liver fibrosis F1-F2), and Group 4 (liver fibrosis F3-F4).

The patients were diagnosed and selected carefully to meet the clinical, biochemical, and sonographical requirements for chronic liver disease. Patients had to meet the following conditions: they were non-obese (BMI <30) and had positive serology for HCV antibody and HCV viremia that detected by PCR technique. Moreover, elastography (Fibroscan) were performed to evaluate the status of fibrosis. On the other hand, some exclusive criteria were taken into consideration. Patients with other causes of liver disease such as HBV and HIV infection, or with severe internal diseases (e.g., cancer, ischemic heart disease, autoimmune disease), and those with chronic liver diseases other than HCV, such as nonalcoholic steatohepatitis, autoimmune hepatitis, biliary disorders, and malignancies were excluded from this study.

The study was approved by the Bioethics Committee of Ain Shams University and was conducted in accordance with the ethical guidelines of Declaration of Helsinki.
The present research followed the guidelines set out by the Medical Ethical Committee of National Hepatology and Tropical Medicine Research Institute, Egypt (Approval No. ITH00114). All HCV samples were provided by the National Hepatology and Tropical Medicine Research Institute. All patients gave written informed consent after explaining the aim and concerns of the study and description of the procedure. Datasheets were coded to ensure anonymity and confidentiality of the patient's data.

\subsection{Biochemical investigations}

All enrolled patients were subjected to complete medical examination to check for the general condition of the liver and other abdominal organs. Moreover, serum samples were collected and stored frozen at -80 ㅇ $\mathrm{C}$ for determination of the studied parameters.

Laboratory investigations were comprised the determination of serum AST and ALT levels, albumin, total, and direct bilirubin (Spectrophotometer Olympus 4000), according to the manufacturers' instructions. Hematological examination: including prothrombin time (PT) and the international normalized ratio (INR) measurement and complete blood counts (CBC) with differential white blood cells counts, were performed by using the auto hematology analyzer.

As previously reported, FIB-4 index could be used similarly to Fibro Test in the diagnosis of advanced fibrosis and cirrhosis of HCV patients ${ }^{[21]}$. The FIB-4 index was determined using the formula: Age (years) X AST [U/I] / (platelets $\left.\left[10^{9} / \mathrm{I}\right] \times(\mathrm{ALT}[\mathrm{U} / \mathrm{I}])^{1 / 2}\right)^{[22]}$. In addition, the AST/ALT ratio (AAR) was also calculated, where the rise in this ratio above 0.8 indicates progressive liver dysfunction, while a ratio $\geq 1$ indicates the incidence of cirrhosis ${ }^{[23]}$.

Moreover, all patients were subjected to determination of the levels of serum SCD163 (Cat. No. DC1630, R \& D Systems, USA), MMP9 (Cat. No. DMP900, R \& D Systems, USA), CK18 (Cat. No. NBP2-75284, Novus biological, USA), and AFP (Cat. No. DAFP00, Novus biological, USA) by using the enzyme linked immunosorbent assay (ELISA) technique according to the manufacturer's instructions.

\subsection{Statistical Analysis}

The results were statistically analyzed using SPSS statistical software version 22.0 (IBM SPSS Statistics). The threshold for statistical significance was set at $p$ value 0.05 . The obtained data were described and compared using frequency and percentages for categorical variables (Pearson chi square), means with standard error (student 
t-test), or median and interquartile range (MannWhitney) for continuous variables.

The potentiality of the selected serum biomarkers to diagnose and differentiate between different stages of liver fibrosis was evaluated using a receiver operating characteristics (ROC) curve.

\section{Results}

\subsection{Indirect biochemical markers for liver fibrosis}

A total of $75 \mathrm{HCV}$ patients (44 males and 31 females) aged from 35 to 60 years, along with age matched 25 normal subjects ( 8 males and 17 females) aged from 38 to 55 years were subjected to various biochemical and hematological analyses. Their biochemical characteristics of all patients are summarized in Table 1.

The results show that the hemoglobin levels recorded no changes in all groups, either when compared to control or Group 2 (F0). Meanwhile, the results reported a significant elevation in WBCs count of Group 2 (F0), and Group 4 (F3-F4), compared to control subjects with no change in Group 3 (F1-F2). In addition, platelets count is significantly decreased in all studied patient groups, compared to control, with a significant decline observed in Group 4 (F3-F4), compared to Group 2 (FO). Besides, INR profile showed a significant elevation in Group 4 (F3-F4), compared to both control and Group 2 (FO).

Moreover, liver function tests; including, AST, ALT, total bilirubin, and direct bilirubin showed significant increment in levels of all these markers in Group 4 (F3F4) only, compared to both control and Group 2 (F0). In the same line, the albumin levels recorded a significant decrement in Group 4 (F3-F4), compared to control group and Group 2 (FO).

Table 1 Biochemical data of all studied groups

\begin{tabular}{lcccc}
\hline $\begin{array}{l}\text { Groups } \\
\text { Parameters }\end{array}$ & $\begin{array}{c}\text { Group 1 } \\
\text { (Control) }\end{array}$ & $\begin{array}{c}\text { Group 2 } \\
\text { (F0) }\end{array}$ & $\begin{array}{c}\text { Group 3 } \\
\text { (F1-F2) }\end{array}$ & $\begin{array}{c}\text { Group 4 } \\
\text { (F3-F4) }\end{array}$ \\
\hline Hemoglobin $(\mathrm{g} / \mathrm{dL})$ & $12.7(11.55-14.5)$ & $11.5(11-13.8)$ & $12.7(11.1-14.5)$ & $12.5(11.05-13.55)$ \\
WBC count $\left(\times 10^{3} / \mathrm{mm}^{3}\right)$ & $4.3(3.6-4.6)$ & $5.4^{\mathrm{a}}(4.05-6.75)$ & $4.5(3.35-6.7)$ & $5.2^{\mathrm{a}}(4.0-7.15)$ \\
Platelets $\left(\times 10^{3} / \mathrm{mm}^{3}\right)$ & $260(237.5-282.0)$ & $181^{\mathrm{a}}(169-237.5)$ & $179^{\mathrm{a}}(147-213.5)$ & $149^{\mathrm{ab}}(134-194)$ \\
INR & $1.0(1.0-1.15)$ & $1.02(1.0-1.10)$ & $1.05(1.0-1.24)$ & $1.2^{\mathrm{ab}}(1.0-1.4)$ \\
AST (U/L) & $20(18-25)$ & $26(20-34)$ & $26(19-36)$ & $48^{\mathrm{ab}}(30-67.5)$ \\
ALT $(\mathrm{U} / \mathrm{L})$ & $24(20.5-29.5)$ & $25(22-35)$ & $37(20-64)$ & $38^{\mathrm{ab}}(24.5-62.5)$ \\
Total bilirubin $(\mathrm{mg} / \mathrm{dL})$ & $0.7(0.6-0.78)$ & $0.78(0.62-0.9)$ & $0.7(0.54-1.1)$ & $1.1^{\mathrm{ab}}(0.8-3.65)$ \\
Direct bilirubin $(\mathrm{mg} / \mathrm{dL})$ & $0.2(0.13-0.21)$ & $0.2(0.12-0.23)$ & $0.2(0.1-0.23)$ & $0.33^{\mathrm{ab}}(0.2-1.05)$ \\
Albumin $(\mathrm{g} / \mathrm{dL})$ & $4.3(3.85-4.55)$ & $4.1(3.6-4.35)$ & $4.1(3.45-4.5)$ & $3.3^{\mathrm{ab}}(2.8-3.85)$ \\
\hline
\end{tabular}

Values are expressed as median (IQR), $p$ value is considered significant $<0.05$ a: significant from control, and b: significant from Group 2.

\subsection{Direct non-invasive markers for liver fibrosis}

The results of the potentiality of the studied noninvasive markers to be used as diagnostic markers for liver fibrosis and to predict the disease progression are represented in Table 2.

As shown in the Table 2, serum sCD163 levels showed a significant decrease in Group 2 (F0), and Group 3 (F1F2), meanwhile, Group 4 (F3-F4) showed a significant increase, compared to control group. Notably, Group 4 (F3-F4) with an advanced stage of fibrosis reported a significant increase in the level of sCD163, compared to non-fibrotic group (Group 2, F0).

Additionally, serum MMP9 levels revealed a significant reduction in both Group 3 (F1-F2), and Group 4 (F3-F4), compared to control group. In advanced fibrosis as illustrated in Group 4 (F3-F4), there was a significant decline in the level of MMP9 compared to non-fibrotic group (Group 2, F0).

On contrary, Ck18 reported remarkable significant elevated levels in all HCV studied patients compared to control subjects' levels. In the same context, a significant rise was documented in the level of CK18 in Group 4 (F3-F4), compared to non-fibrotic HCV group (Group 2, F0).

AFP levels, as a gold standard marker for liver fibrosis and carcinoma, were also measured to judge the efficiency of the current studied markers. AFP levels recorded a significant increase in all studied HCV patients, compared to control normal group. Similar to SCD136, and CK18, with dissimilar to MMP9, the level of AFP was significantly augmented in advanced fibrotic group [Group 4 (F3-F4)], compared to non-fibrotic group [Group 4 (F3-F4)]. 
Table 2 Direct non-invasive markers for liver fibrosis

\begin{tabular}{lcccc}
\hline Groups & Group 1 & Group 2 & Group 3 & $\begin{array}{c}\text { Group 4 } \\
\text { Parameters }\end{array}$ \\
(Control) & (F0) & (F1-F2) & (F3-F4) \\
\hline sCD163 $(\mathrm{ng} / \mathrm{ml})$ & $631.0(502.5-743.0)$ & $415^{\mathrm{a}}(294-681.5)$ & $497^{\mathrm{a}}(406-655)$ & $837^{\mathrm{ab}}(765-937)$ \\
MMP9 $(\mathrm{ng} / \mathrm{ml})$ & $1786(1316.0-9030.5)$ & $1240(510-3934.5)$ & $800^{\mathrm{a}}(454.5-1295.5)$ & $472^{\mathrm{ab}}(208.5-1023.5)$ \\
CK18 $(\mathrm{mlu} / \mathrm{ml})$ & $13.2(5.75-31.35)$ & $27.7^{\mathrm{a}}(19.75-78.15)$ & $36.1^{\mathrm{a}}(19.1-51.6)$ & $44.1^{\mathrm{a}}(29.8-96.95)$ \\
AFP $(\mathrm{ng} / \mathrm{ml})$ & $2.1(1.35-3.30)$ & $2.7(2.0-3.7)$ & $3.5^{\mathrm{a}}(2.7-4.35)$ & $7.0^{\mathrm{ab}}(4.65-11.3)$ \\
\hline
\end{tabular}

Values are expressed as median (IQR), $p$ value is considered significant $<0.05^{\text {a }}$ : significant from control, and ${ }^{\text {b: }}$ significant from Group 2.

\subsection{Indirect non-invasive diagnostic markers of liver fibrosis}

Several scores have been suggested in an effort to avoid the sampling of a liver biopsy in the diagnosis and management of fibrosis in chronic liver disease. FIB-4 index is a calculated fibrotic score based on different routine biochemical markers. As shown in Table 3, FIB-4 index was increased in a highly significant manner compared to control [83\%, 84\%, and 306\% for Group 2 (F0), Group 3 (F1-F2), and Group 4 (F3-F4), respectively].

Furthermore, AAR showed a significant increase in Group 2 and 3 (47\% and 57\%, respectively), with enlarged increase with fibrosis progression as observed in advanced fibrotic group (132\%).

3.4 Validation of the studied serum markers for diagnosis of liver fibrosis

Comparative ROC analysis of the selected diagnostic markers is summarized in Figs. 1-3. The area under the ROC curve (AUC) is used to assess the efficacy of the tested serum direct biomarkers for predicting extensive liver fibrosis. The results obtained showed that the studied serum markers SCD136, MMP9, and CK18 are very specific, and highly sensitive. The combined evaluation of SCD163, CK18 and MMP9 levels at the cut off 0.745 showed high discrimination [AUC 0.818 (95\% Cl: 0.672-0.965; $p<0.001)$ ], sensitivity $60 \%$, and specificity $85 \%$ in the diagnosis of $\mathrm{HCV}$ infected patients Fig. 1. Also, this triple marker panel showed high performance to discriminate $\mathrm{HCV}$ infected patients with lower grade of fibrosis from the patients without fibrosis at the cut off 0.745 showed high discrimination [AUC 0.770 (95\% Cl: 0.637-0.902; $p<$ $0.001)$ ], sensitivity $60 \%$, and specificity $76 \%$ Fig. 2 . To diagnose the patients with severe fibrosis, the panel can discriminate the patients with the highest performance to discriminate patients at the cut off 0.745 [(AUC 0.986 (95\% Cl: 0.960-1.000; $p<0.001$ )], sensitivity $92 \%$, and specificity $100 \%$ Fig. 3. Such observations suggest that there is an association between SCD163, CK18, and MMP9 levels for the fibrosis progression in HCV infected patients.

Table 3 FIB-4 and AAR as direct non-invasive markers for liver fibrosis

\begin{tabular}{|c|c|c|c|c|c|}
\hline $\begin{array}{l}\text { Groups } \\
\text { Parameters }\end{array}$ & & $\begin{array}{l}\text { Group } 1 \\
\text { (Control) }\end{array}$ & $\begin{array}{c}\text { Group } 2 \\
\text { (F0) }\end{array}$ & $\begin{array}{l}\text { Group } 3 \\
\text { (F1-F2) }\end{array}$ & $\begin{array}{c}\text { Group } 4 \\
\text { (F3-F4) }\end{array}$ \\
\hline \multirow{3}{*}{ FIB-4 } & Mean \pm SE & $0.550 \pm 0.4$ & $0.95 \pm 0.1$ & $0.99 \pm 0.2$ & $2.10 \pm 0.3$ \\
\hline & $\%$ change & & $83 \%$ & $84 \%$ & $306 \%$ \\
\hline & $p$ value & & $<0.001$ & $<0.001$ & $<0.001$ \\
\hline \multirow{3}{*}{ AAR } & Mean \pm SE & $0.59 \pm 0.07$ & $0.88 \pm 0.07$ & $0.98 \pm 0.08$ & $1.36 \pm 0.5$ \\
\hline & $\%$ change & & $47 \%$ & $57 \%$ & $132 \%$ \\
\hline & $p$ value & & $<0.01$ & $<0.01$ & $<0.001$ \\
\hline
\end{tabular}




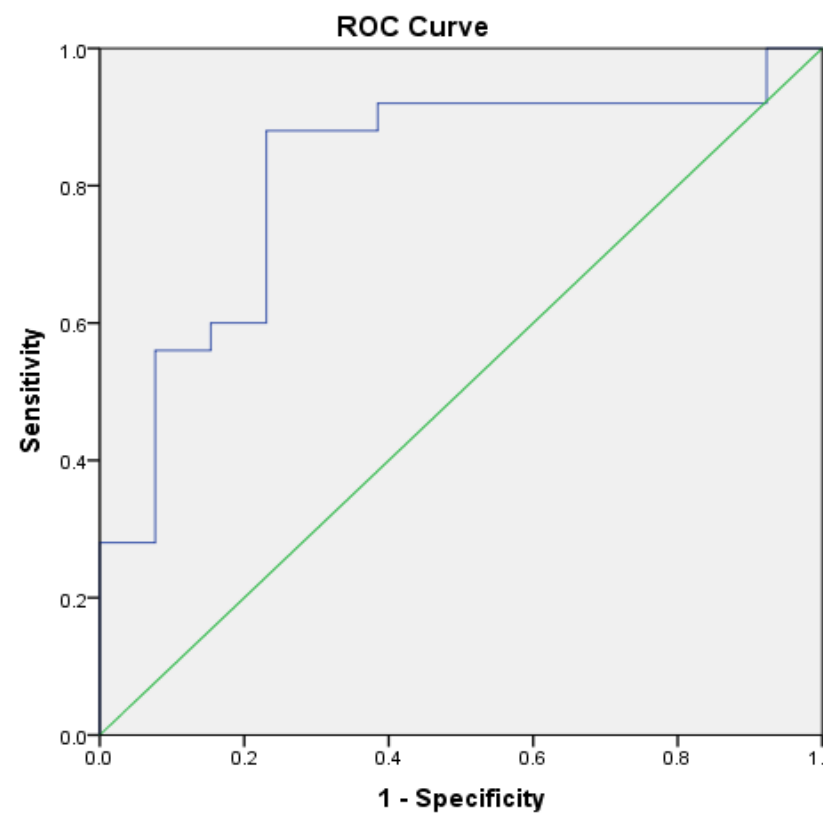

Fig. 1 Combined ROC curve of SCD163, MMP9, and CK18 in Group 2 vs Group 1

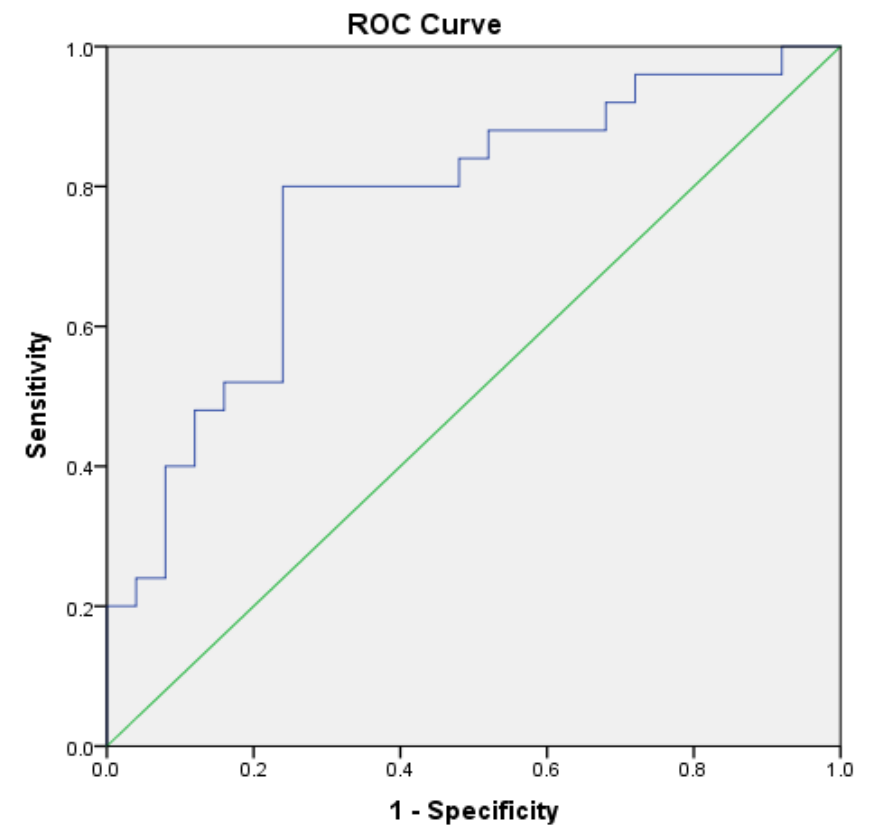

Fig. 2 Combined ROC curve of sCD163, MMP9, and CK18 in Group 3 vs Group 2

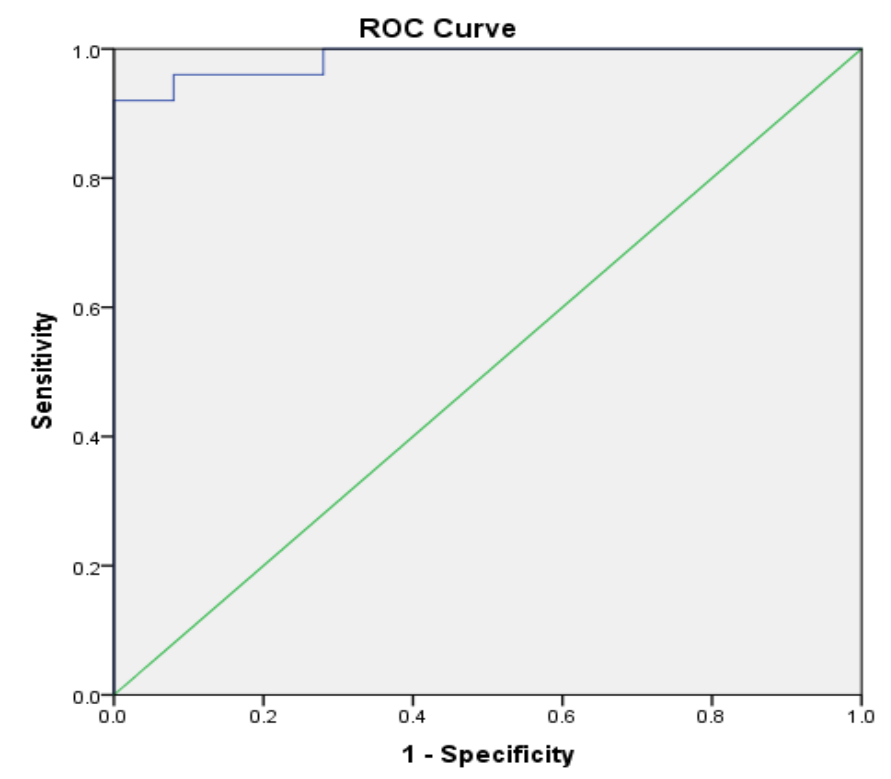

Fig. 3 Combined ROC curve of sCD163, MMP9, and CK18 in Group 4 vs Group 2

\section{Discussion}

$\mathrm{HCV}$ is one of the most common causes of chronic hepatitis, liver cirrhosis, and hepatocellular carcinoma (HCC). About 55$85 \%$ of HCV-infected patients develop chronic active HCV and go through the process of developing fibrosis, cirrhosis, and may progress to decompensated cirrhosis and HCC ${ }^{[24]}$. Liver fibrosis has to be the most important predictor of liver-related complications such as portal hypertension, decompensation events, cancer, and death ${ }^{[25]}$.
Liver biopsy, as the standard analysis for liver fibrosis, has several limitations. So, different biochemical serum markers for diagnosis and prediction of liver fibrosis have been extensively studied as well as histology at predicting clinical outcomes. They could be divided into direct fibrosis markers, including matrix-specific molecules and immunological markers/cytokines, and indirect fibrosis markers (such as liver function tests and platelets) ${ }^{[26]}$. 
Non-invasive, inexpensive tests to diagnose and monitor fibrosis in patients with chronic liver disease are desperately needed to overcome the disadvantages of liver biopsy samples and late diagnosis issues. Since blood samples are readily available, looking for serum markers that can replace liver biopsies or measures of liver stiffness is appealing ${ }^{[26]}$.

Kupffer cells are hepatic macrophages that play a key role in liver inflammation and fibrosis, account for more than $80 \%$ of tissue macrophages, and express higher levels of CD163. Given these characteristics, it's possible that serum levels of SCD163 over-reflect chronic hepatic inflammation [27]. Serum levels of sCD163 have been shown to be elevated in a variety of inflammatory diseases, such as liver diseases, diabetes, and infectious diseases ${ }^{[28]}$. It has also been associated with inflammation, specifically the hepatic activity index, and fibrosis ${ }^{[29]}$. Furthermore, increased SCD163 levels are significantly related to portal hypertension, Child-Pugh score, and model for end-stage liver disease score in patients with cirrhosis of various etiologies ${ }^{[30]}$.

The data from the current studies revealed that the serum level of sCD163 was significantly lower in the HCV-infected patients without and with low grade fibrosis, peaking in the advanced stage of liver fibrosis in comparison to control subjects and non-fibrotic $\mathrm{HCV}$ patient groups $(p<0.001)$. This outcome is similar to that of the previous finding ${ }^{[31]}$, which stated that the serum SCD163 levels are elevated in patients with chronic HCV patients, reflecting hepatic macrophage activation. The sCD163 levels may be used as a useful diagnostic tool to monitor the progression of liver fibrosis in the management of chronic HCV patients. Similarly, two previous studies also reported that sCD163 levels are known to be elevated in chronic HBV infection, and the results of these studies reported a strong correlation between histological inflammation, fibrosis, and SCD163 levels ${ }^{[28,32]}$. These data are in accordance with the results of the current study.

CK18 is originally expressed by hepatocytes. Infected hepatocytes with HCV are a major source of high expression levels of the intermediate filament protein CK18, which accumulates rapidly in the circulation through both apoptosis and necrosis, and triggers the inflammatory condition, regeneration, and liver fibrosis ${ }^{[33,34]}$.
Serum CK18 levels were found to be significantly higher in all studied groups of the present study $(p<$ 0.001) compared to the control group, with no significant elevation in fibrotic groups compared to the non-fibrotic (FO) group. These findings are in agreement with other prior studies ${ }^{[19]}$, which stated that the development/progression of non-alcoholic fatty liver disease (NAFLD) and HCV-related fibrosis/steatosis are strongly related to serum CK18 levels. Several studies have proved the improvement in diagnostic yield in the case of fibrotic HCV patients when the CK18 test is combined with other noninvasive modalities. Accordingly, El Zefzafy et al. [35] reported that patients with chronic HCV infection have significantly higher serum CK18 levels, with HCC patients having the highest levels, which may be related to the disease's pathophysiology. Serum CK18 levels in chronic HCV infection, liver cirrhosis, and HCC patients can be used to monitor disease activity.

The combination of AFP and CK18 increased the sensitivity of HCC detection. Moreover, another study provided the evidence for macrophage activation in human NAFLD and non-alcoholic steatohepatitis (NASH), with associations between SCD163 and pathological liver disease stages. The study stated that the positive correlation between SCD163 and CK18 recommends the incidence of apoptotic events which may be contributed to macrophage activation in various liver diseases. So, sCD163 combined with CK18 could be used as good biochemical markers for predicting advanced fibrosis and may have potential as potential biomarkers for the non-invasive assessment of disease stage ${ }^{[36]}$.

In addition, MMPs are well-known to be involved in various stages of liver diseases ranging from liver injury, inflammation, fibrosis, cirrhosis, and incidence of hepatocellular carcinoma to disease resolution and liver renewal ${ }^{[37]}$. The exact mechanism of action of MMPs in different liver disorders is largely unknown. However, information from various experimental studies indicates that these MMPs could control several cellular activities, including proliferation and survival, gene expression, as well as multiple aspects of inflammation ${ }^{[38]}$. The current findings revealed that serum MMP9 levels were significantly decreased in all studied HCV groups in comparison to controls and also in the severely advanced fibrotic group (Group 4) 
compared to the non-fibrotic group (Group 2) ( $p<$ $0.001)$. This decrease is contradictory to what was reported previously, which stated that MMP9 serum levels were significantly increased in chronic HCV patients than in controls. In addition, MMP2 was elevated during all the stages of alcoholic liver cirrhosis, while MMP8 and MMP9 were only increased in advanced liver cirrhosis ${ }^{[39]}$. In correspondence, other studies found that serum MMP9 levels in patients with chronic HCV were significantly lower than in healthy controls ${ }^{[40]}$. This variability in results may be related to differences in study populations, type, and severity of chronic liver disease, and differences in assay systems. Based on previous data, the combination of MMP9, AFP, infiltrated leukocytes, AST/ALT ratio, and platelet count has been suggested for F2-F4 staging of fibrosis in patients with chronic HCV infection ${ }^{[41]}$.

A previous study reported the correlation between the higher levels of CK18 and MMP9 in chronic HBV patients compared to healthy controls, and this correlation was associated with hepatic fibrosis and cirrhosis ${ }^{[42] .}$

AFP is mostly associated with the presence of HCC, but there is a lot of evidence that proves its utility in diagnosing other liver conditions such as fibrosis ${ }^{[43]}$, and even other than liver diseases such as down syndrome ${ }^{[44]}$. The relation between AFP elevation in the serum and the progression of liver fibrosis has been proven, especially in chronic HCV patients ${ }^{[45]}$. The current findings revealed a positive association between the serum level of AFP and the Fibroscan stage of hepatic fibrosis. These results are in agreement with the results of Attallah et al. ${ }^{[46]}$ who recorded the highly significant positive association between AFP serum levels and the stage of hepatic fibrosis in chronic HCV patients without HCC.

When compared to the normal control, the serum level of AFP was increased significantly in fibrotic groups. Furthermore, it is significantly increased in the severely fibrotic group (Group 4) in comparison to patients without fibrosis (Group 2). This rise correlated with the progression of fibrosis grades, suggesting that AFP could be one of the low-cost routine laboratory tests that predict the rate of fibrosis progression. As a result, early detection is important for increasing patient survival rates.
In the current study, AUC or combined ROC curves were used to determine the most indicative serum biomarker combination for predicting extensive liver fibrosis. With the progression of fibrosis, the performance of both direct and indirect serum markers improved, according to the results of present findings. The current data proved that the selected panel of CD163, CK18, and MMP9 has high performance to differentiate HCV patients from healthy subjects. Moreover, the triple panel has the highest performance in discriminating patients with severe fibrosis from HCV-infected patients without fibrosis. The results indicate the importance of this triple panel in predicting disease progression.

\section{Conclusion}

The current data supported that MMP9 was a more specific biomarker for stages of fibrosis (F1 and F2), whereas serum SCD163 was a more specific biomarker for stages of fibrosis (F3 and F4).

\section{References}

1. Zedan, H. E., Talaat, R. M., Ammar, A. A. A. and Sakr, M. A. (2019). Interferon-Lambda 4 Gene Polymorphisms Predict Treatment Response in Egyptian HCV Genotype 4 Patients Exposed to Radiation. Egypt. J. Hosp. Med., 77(1): $4748-453$.

2. Abd-Elsalam, S., Sharaf-Eldin, M., Soliman, S., Elfert, A., Badawi, R. and Ahmad, Y. K. (2018). Efficacy and safety of sofosbuvir plus ribavirin for treatment of cirrhotic patients with genotype 4 hepatitis $C$ virus in real-life clinical practice. Arch. Virol., 163(1): $51-56$.

3. Khatun, M. and Ray, R. B. (2019). Mechanisms Underlying Hepatitis C Virus-Associated Hepatic Fibrosis. Cells, 8(10): 1249.

4. Koyama, Y. and Brenner, D. A. (2017). Liver inflammation and fibrosis. J. Clin. Invest., 127(1): $55-64$.

5. Baskic, D., Vukovic, V., Popovic, S., Jovanovic, D., Mitrovic, S., Djurdjevic, P. and et al. (2019). Chronic hepatitis C: Conspectus of immunological events in the course of fibrosis evolution. PLoS One, 14(7): e0221142.

6. Goodman, Z. D. (2007). Grading and staging systems for inflammation and fibrosis in chronic liver diseases q. J. Hepatol., 47(2007): $598-607$. 
7. Shipley, L. C., Axley, P. D. and Singal, A. K. (2019). Liver Fibrosis: A Clinical Update. Eur. Med. J. Hepatol., 7(1): $105-117$.

8. Machado, N. O. (2011). Complications of Liver Biopsy - Risk Factors, Management and Recommendations. Takahashi, H. (ed). Liver Biopsy, $393-404$.

9. De Lédinghen, V. and Vergniol, J. (2008). Transient elastography (FibroScan) Élastographie impulsionnelle (FibroScan). Gastroentérologie. Clin. Biol., 32: 58 - 67.

10. Soresi, M., Giannitrapani, L., Cervello, M., Licata, A. and Montalto, G. (2014). Non invasive tools for the diagnosis of liver cirrhosis. World J. Gastroenterol., 20(48): 18131 - 18150 .

11. Neuberger, J. and Cain, O. (2021). The Need for Alternatives to Liver Biopsies: Non-Invasive Analytics and Diagnostics. Hepatic Med. Evid. Res., 13: $59-69$.

12. Lurie, Y., Webb, M., Cytter-Kuint, R., Shteingart, S. and Lederkremer, G. Z. (2015). Non-invasive diagnosis of liver fibrosis and cirrhosis. World J. Gastroenterol., 21(41): 11567.

13. Kristiansen, M., Graversen, J. H., Jacobsen, C., Sonne, O., Hoffman, H. J., Law, S. K. A. and et al. Identification of the haemoglobin scavenger receptor. Nature, 409(6817): 198 201.

14. Gantzel, R. H., Kjær, M. B., Laursen, T. L., Kazankov, K., George, J., Møller, H. J. and et al. (2021). Macrophage Activation Markers, Soluble CD163 and Mannose Receptor, in Liver Fibrosis. Front Med., 7: 1074.

15. Björklund, J., Laursen, T. L., Sandahl, T. D., Møller, H. J., Vilstrup, H., Ott, P. and et al. (2018). High hepatic macrophage activation and low liver function in stable Wilson patients - a Danish cross-sectional study. Orphanet. J. Rare Dis., 13(1): $169-175$.

16. Roeb, E. (2018). Matrix metalloproteinases and liver fibrosis (translational aspects). Matrix Biol., 68-69: 463 - 473.
17. Pardo, A., Cabrera, S., Maldonado, M. and Selman, M. (2021). Role of matrix metalloproteinases in the pathogenesis of idiopathic pulmonary fibrosis. Respir. Res., 17(1).

18. Martinez-Castillo, M., Hernandez-Barragan, A., Flores-Vasconcelos, I., Galicia-Moreno, M., Rosique-Oramas, D., Perez-Hernandez, J. L. and et al. (2021). Production and activity of matrix metalloproteinases during liver fibrosis progression of chronic hepatitis C patients. World J. Hepatol., 13(2): 218.

19. Darweesh, S. K., Abdelaziz, R. A., Abd-Elfatah, D. S., Abdelazim, N. A., Fathi, S. A., Attia, D. and et al. (2019). Serum cytokeratin-18 and its relation to liver fibrosis and steatosis diagnosed by FibroScan and controlled attenuation parameter in nonalcoholic fatty liver disease and hepatitis $C$ virus patients. Eur. J. Gastroenterol. Hepatol., 31(5): 633 - 641.

20. Kosasih, S., Zhi Qin, W., Abdul Rani, R., Abd Hamid, N., Chai Soon, N., Azhar Shah, S. and et al. (2018). Relationship between Serum Cytokeratin-18, Control Attenuation Parameter, NAFLD Fibrosis Score, and Liver Steatosis in Nonalcoholic Fatty Liver Disease. Int. J. Hepatol., 2018.

21. Vallet-Pichard, A., Mallet, V., Nalpas, B., Verkarre, V., Nalpas, A., Dhalluin-Venier, V. and et al. (2021). FIB-4: An inexpensive and accurate marker of fibrosis in HCV infection. comparison with liver biopsy and fibrotest. Hepatology, 46(1): $32-36$.

22. Sterling, R. K., Lissen, E., Clumeck, N., Sola, R., Correa, M. C., Montaner, J. and et al. (2003). Development of a simple noninvasive index to predict significant fibrosis in patients with HIV/HCV coinfection. Hepatology, 43(6): 1317-1325.

23. Giannini, E., Risso, D., Botta, F., Chiarbonello, B., Fasoli, A., Malfatti, F. and et al. (2003). Validity and clinical utility of the aspartate aminotransferasealanine aminotransferase ratio in assessing disease severity and prognosis in patients with hepatitis $C$ virus-related chronic liver disease. Arch. Intern. Med., 163(2): 218 - 224. 
24. Axley, P., Ahmed, Z., Ravi, S. and Singal, A. K. (2018). Hepatitis C Virus and Hepatocellular Carcinoma: A Narrative Review. J. Clin. Transl. Hepatol., 6(1): 79.

25. Irvine, K. M., Wockner, L. F., Shanker, M., Fagan, K. J., Horsfall, L. U., Fletcher, L. M. and et al. (2021). The Enhanced liver fibrosis score is associated with clinical outcomes and disease progression in patients with chronic liver disease. Liver Int., 36(3): 370 - 377.

26. Castera, L. (2012). Noninvasive methods to assess liver disease in patients with hepatitis B or C. Gastroenterology, 142(6): 1293 1302.e4.

27. Mueller, J. L., Feeney, E. R., Zheng, H., Misdraji, J., Kruger, A. J., Alatrakchi, N. and et al. (2015). Circulating Soluble CD163 is Associated with Steatohepatitis and Advanced Fibrosis in Nonalcoholic Fatty Liver Disease. Clin. Transl. Gastroenterol., 6(10): e114.

28. Andersen, E., Rødgaard-Hansen, S., Moessner, B., Christensen, P., Møller, H. and Weis, N. (2014). Macrophage-related serum biomarkers soluble CD163 (sCD163) and soluble mannose receptor (sMR) to differentiate mild liver fibrosis from cirrhosis in patients with chronic hepatitis C: a pilot study. Eur. J. Clin. Microbiol. Infect. Dis., 33(1): $117-122$.

29. Kazankov, K., Barrera, F., Møller, H. J., Bibby, B. M., Vilstrup, H., George, J. and et al. (2014). Soluble CD163, a macrophage activation marker, is independently associated with fibrosis in patients with chronic viral hepatitis B and C. Hepatology, 60(2): $521-530$.

30. Sakr, M. A., Mohamed, K. A. H., Hussein, A. M., Fouad, M. H., Allam, A. S. and Safwat, E. (2021). Diagnostic and prognostic value of serum soluble CD163 in cirrhotic patients with hepatitis $C$ virus-related hepatocellular carcinoma before and after locoregional therapy. Egypt. Liver J., 11(1): 1 - 10.
31. Zhao, S. X., Li, W. C., Fu, N., Kong, L. B., Zhang, Q. S., Han, F. and et al. (2021). CD14+ monocytes and $\mathrm{CD} 163+$ macrophages correlate with the severity of liver fibrosis in patients with chronic hepatitis C. Exp. Ther. Med., 20(6): 1-11.

32. Dultz, G., Gerber, L., Farnik,H., Berger, A., Vermehren, J., Pleli, T. and et al. (2015). Soluble CD163 is an indicator of liver inflammation and fibrosis in patients chronically infected with the hepatitis B virus. J. Viral Hepat., 22(4): 427-432.

33. Abdel Haleem, H., Zayed, N., Abdel Hafez, H., Fouad, A., Akl, M., Hassan, M. and et al. (2013). Evaluation of the diagnostic value of serum and tissue apoptotic cytokeratin-18 in patients with chronic hepatitis C. Arab J. Gastroenterol., 14(2): $68-72$.

34. Yilmaz Y. (2021). Systematic review: caspasecleaved fragments of cytokeratin 18 - the promises and challenges of a biomarker for chronic liver disease. Aliment Pharmacol. Ther., 30(11-12): $1103-1109$.

35. El-Zefzafy, W., Eltokhy, H., Mohamed, N. A. E. G. and Abu-Zahab, Z. (2015). Significance of Serum Cytokeratin-18 in Prediction of Hepatocellular Carcinoma in Chronic Hepatitis C Infected Egyptian Patients. Maced. J. Med. Sci., 3(1): 117 123.

36. Kazankov, K., Barrera, F., Møller, H. J., Rosso, C., Bugianesi, E., David, E. and et al. (2016). The macrophage activation marker sCD163 is associated with morphological disease stages in patients with non-alcoholic fatty liver disease. Liver Int., 36(10): 1549 - 1557.

37. Geervliet, E. and Bansal R. (2021). Matrix Metalloproteinases as Potential Biomarkers and Therapeutic Targets in Liver Diseases. Cells, 2(5): 1212.

38. Naim, A., Pan, Q., Baig, M. S. (2017). Matrix Metalloproteinases (MMPs) in Liver Diseases. J. Clin. Exp. Hepatol., 7(4):367.

39. Prystupa, A., Boguszewska-Czubara, A., BojarskaJunak, A., Toruń-Jurkowska, A., Roliński, J. and Załuska, W. (2015). Activity of MMP-2, MMP-8 and MMP-9 in serum as a marker of progression of alcoholic liver disease in people from Lublin Region, eastern Poland. Ann. Agric. Environ. Med., 22(2): $325-328$. 
40. Leroy, V., Monier, F., Bottari, S., Trocme, C., Sturm, N., Hilleret, M. N. and et al. (2004). Circulating Matrix Metalloproteinases 1, 2, 9 and Their Inhibitors TIMP-1 and TIMP-2 as Serum Markers of Liver Fibrosis in Patients with Chronic Hepatitis C: Comparison with PIIINP and Hyaluronic Acid. Am. J. Gastroenterol., 99(2): 271 - 279.

41. Attallah, A. M., Omran, D., Omran, M. M., Albannan, M. S., Zayed, R. A., Saif, S. and et al. (2018). Fibro-Mark: a panel of laboratory parameters for predicting significant fibrosis in chronic hepatitis $\mathrm{C}$ patients. Br. J. Biomed. Sci., 75(1): $19-23$.

42. Sumer, S., Demir, N. A., Kölgelier, S., Inkaya, A. C., Arpaci, A., Demir, L. S. and et al. (2013). The clinical significance of serum apoptotic cytokeratin 18 neoepitope M30 (CK-18 M30) and matrix metalloproteinase 2 (MMP-2) levels in chronic hepatitis B patients with cirrhosis. Hepat. Mon., 13(6).
43. Liu, Y., Lin, B., Zeng, D., Zhu, Y., Chen, J., Zheng, Q. and et al. (2014). Alpha-fetoprotein level as a biomarker of liver fibrosis status: a cross-sectional study of 619 consecutive patients with chronic hepatitis B. BMC Gastroenterol., 14(1): $1-8$.

44. Li, Y., Zhang, X., Hong, D., Guan, X., Lv, S., Sun, Y. and et al. (2018). Significance of data analysis in the quality control of prenatal screening for down syndrome. Biomed. Reports, 8(5): $447-453$.

45. Hu, K. Q., Kyulo, N. L., Lim, N., Elhazin, B., Hillebrand, D. J. and Bock, T. (2004). Clinical significance of elevated alpha-fetoprotein (AFP) in patients with chronic hepatitis $C$, but not hepatocellular carcinoma. Am. J. Gastroenterol., 99(5): 860-865.

46. Attallah, A. M., Omran, M. M., Nasif, W. A., Ghaly, M. F., El-Shanshoury, A. E. R. R., Abdalla, M. S. and et al. (2012). Diagnostic Performances of Hepatitis C Virus-NS4 Antigen in Patients with Different Liver Pathologies. Arch. Med. Res., 43(7): 555 - 562. 